\title{
SalivaPrint as a Non-invasive Diagnostic Tool
}

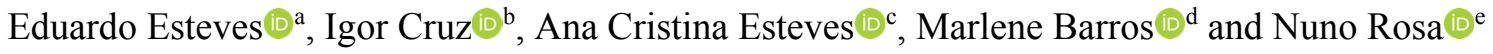 \\ Universidade Católica Portuguesa, Faculty of Dental Medicine, \\ Center for Interdisciplinary Research in Health (CIIS), Portugal
}

Keywords: $\quad$ Saliva Diagnostic, Saliva Protein Profile, Machine Learning Strategies, Health Diagnostic.

\begin{abstract}
Currently, the molecular diagnosis is based on the quantification of RNA, proteins and metabolites because they present changes in their quantity related to clinical situations. The same molecules are not generally suitable for early diagnosis or to follow clinical evolution, making necessary strategies to evaluate the complete molecular scenario. There are already experimental strategies that allow the determination of total protein profiles from saliva samples (the SalivaPrint). The goal of this work is to identify a profile of saliva proteins (similar to a fingerprint) and, using computational methods, identify how this profiles changes with age and gender. So far it has been possible to collect 79 samples as well as the metadata associated with each sample using an electronic questionnaire developed by us. A total protein profile was obtained and their association with gender was verified using statistical methods. Currently we are developing the Python scripts for automatic data acquiring and normalization. Total protein profiles annotation on a database (SalivaPrintDB) and their integration with the factors that affects them using machine learning strategies can empower the use of the approach proposed on this work as a tool for monitoring the individual's health status.
\end{abstract}

\section{INTRODUCTION}

In the last decade, saliva has been studied as a noninvasive, easily obtainable fluid, with diagnosis potential (Loo et al., 2010).

Saliva reflects the secretions of the 3 largest salivary glands (parotid, submandibular and sublingual), smaller salivary glands, crevicular fluid and also contains serum components, transported by blood capillaries and subsequently transferred by diffusion, transport and/or ultrafiltration. The presence of serum proteins in saliva enhances its use as a systemic health status monitoring tool (Castagnola et al., 2017; Kaczor-Urbanowicz et al., 2017; Kaushik and Mujawar, 2018; Wang, KaczorUrbanowicz, and Wong, 2017).

We recently described an automated capillary electrophoresis-based strategy that allows to obtain a salivary protein profile - the SalivaPrint Toolkit (Cruz et al., 2018).
Since proteins are separated according to their molecular mass, changes in peak morphology or fluorescence intensity (translated by changes in peak height) correspond to changes in the amount of proteins or in the type of proteins being expressed. The association of clinical and personal data to saliva protein signatures, the SalivaPrint, to alterations in different health/disease situations, will allow to build a powerful framework for the creation of noninvasive diagnostic strategies.

Preliminary results of data extraction (SalivaPrint-Toolkit, Cruz et al., 2018) from capillary electrophoresis allowed to identify the potential for the creation of a SalivaPrint database.

It is known that some factors such as age, sex, or circadian rhythm (Castagnola et al., 2011, 2017; Murr et al., 2017), contribute to altering the expression of salivary proteins . It is essential to define the signature of proteins correspondent to the "healthy individual", and simultaneously consider the intra-individual variations.

\footnotetext{
a(D) https://orcid.org/0000-0001-5458-4978

b(iD https://orcid.org/0000-0002-7082-297X

c(iD) https://orcid.org/0000-0003-2239-2976

d(D) https://orcid.org/0000-0003-0631-4062

e (iD https://orcid.org/0000-0003-4604-0780
} 
Additionally, will be useful to identify potential proteins that could reflect the changes in the SalivaPrint profiles characteristic of different situations. Data on proteins in saliva associated with disease and health is already extensive. Our group compilated this information in SalivaTecDB database (http://salivatec.viseu.ucp.pt/salivatec-db, Arrais et al., 2013; Rosa et al., 2012), which is an important support for the identification of proteins that may potentially be associated with certain signatures. SalivaTecDB has currently stored more than 4000 human salivary proteins and is constantly being updated.

The profile of salivary proteins, expressed in different situations, has the potential of being used for noninvasive diagnosis. The objectives of this work are:

1- Establish the Health-SalivaPrint(s). The factors that influence the protein profile of saliva in healthy individuals will be considered.

2- Build a SalivaPrint database. These profiles will posteriorly be used for the development of a tool for heath monitoring using machine learning strategies.

\section{MOTIVATION}

Nowadays, molecular diagnosis is based on the quantification of RNA, proteins or metabolites whose concentration can be correlated to clinical situations. Usually, these molecules are not suitable for early diagnosis or to follow clinical evolution. Therefore, strategies to evaluate the complete molecular scenario - early diagnosis, diagnosis and clinical evolution are necessary.

The potential of proteins for a large-scale diagnosis depends on cheap and preferably noninvasive strategies for screening. Bioinformatics strategies and solutions to work on different types of data: from biological related data to personal and clinical information's, is the best approach. Data integration by these methods is an asset to predict the pathological status before clinical outcomes.

This work explores the potential of saliva protein profile obtained by capillary electrophoresis - the SalivaPrint - as a strategy to obtain signatures of health/disease states. The application of computational methods (machine learning strategies) will allow the establishment of a signature that characterizes the healthy individual and how it varies with diverse factors.
At this time, we are only interested in establishing the entire SalivaPrint methodology, from capillary electrophoresis to determining which factors influence the protein profile (of healthy individuals) including the creation of the database that stores all this information.

\section{METHODOLOGY}

This study was approved by the Ethics Committee of the Portuguese Catholic University (UCP). In addition, donors will be asked, prior to any collection, the consent for samples' collection. All work will be carried out in accordance with the principles of the Helsinki declaration, as reviewed in 2008.

In order to achieve the proposed objectives, the strategy presented in figure 1 will be followed:

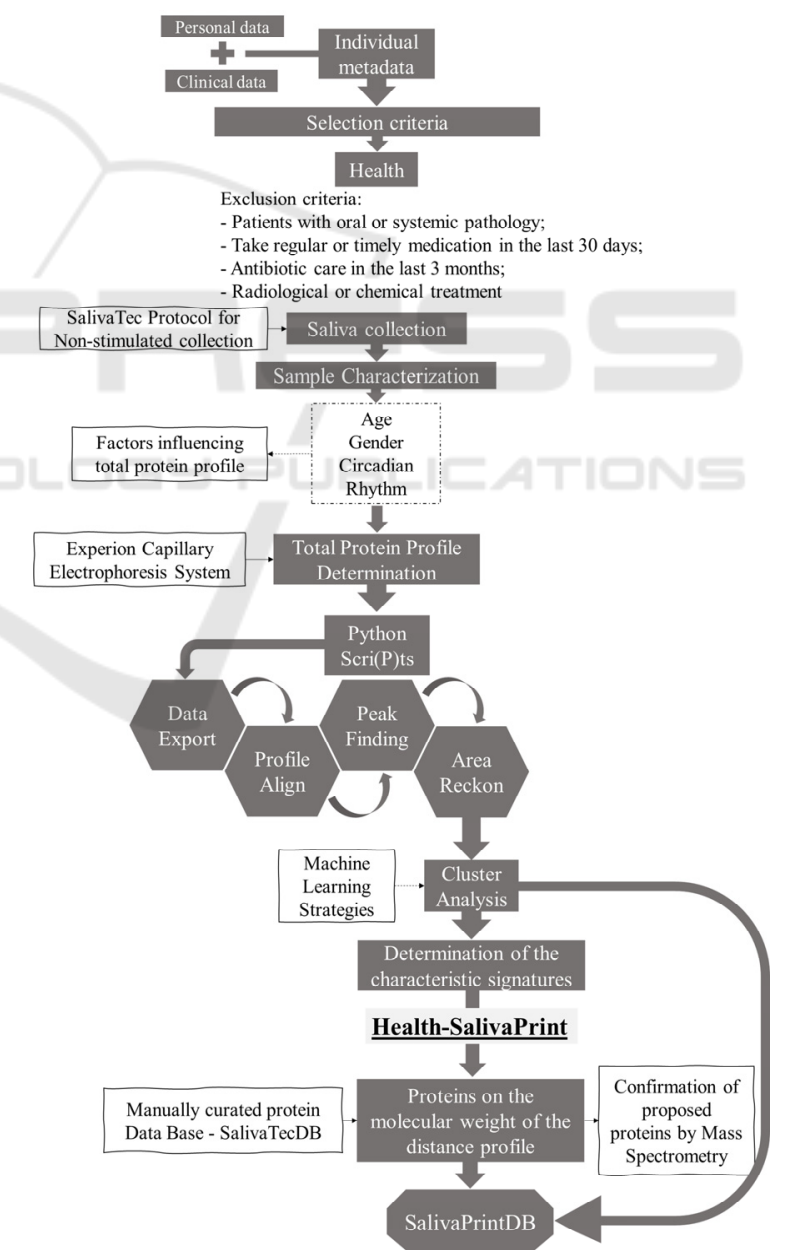

Figure 1: Flowchart of proposed strategy plan. 


\subsection{Questionnaire Preparation to Characterize the Biological Sample}

An online filling questionnaire will be created using Qualtrics ${ }^{\circledR}$ software, for the collection of clinical and personal information.

Questions will be defined to address the individual's personal data and clinical status (SalivaTec Donor Form). Each donor will be observed by a dentist (collaboration with the Dental Clinic of UCP), for oral health status evaluation, taking into account the PSR index (Periodontal Screening Recording) and DMFT (Decayed, Missing, Filled Teeth) (Anón, 2002; Schuller and Holst, 2001).

\subsection{Collection and Processing of Saliva Samples}

To define the protein profile of healthy individuals, samples of non-stimulated saliva will be collected, using an established protocol (Rosa et al., 2016), from patients from the dental clinic of the Portuguese Catholic University and from seniors in the Senior Activity program from the municipality of Viseu. The criteria for exclusion of healthy donors were defined according to the literature: patients with oral or systemic pathology; intake of regular or punctual medication in the last 30 days; antibiotic administration in the last 3 months; never subjected to radiological or chemical treatment (Mozaffari et al., 2019; Murr et al., 2017; Wang et al., 2015). Samples will be categorized into groups of different ages and genders.

These collections will be made under partnerships already established with each institution.

Total protein concentration, $\mathrm{pH}$ and volume of each sample will be determined.

\subsection{Determination of the Total Profile of Proteins by Capillary Electrophoresis}

Saliva protein profile (SalivaPrint) will be determined by capillary electrophoresis, using the Experion ${ }^{\mathrm{TM}}$ Automated Electrophoresis System (BioRad) in standard protein chips (Experion ${ }^{\mathrm{TM}}$ Pro260 Analysis Kit). The samples will be analyzed according to the technical specifications provided by BioRad.

Protein profile and the quantification of bands will be obtained using the Experion ${ }^{\mathrm{TM}}$ Software, version 3.20.

\subsection{Determination of Factors Influencing Health-SalivaPrint}

The clustering of profiles will be tested according to potentially influencing factors of salivary proteome [age, gender and circadian rhythm (Castagnola et al., 2017), among others].

For this, a variety of statistical analysis models (e.g. Kruskall-Wallis) will be applied for comparing each profile between influence groups created. Also, data classification techniques will be implemented with high robustness and effectiveness, such as Random Forest, as well as approaches based on unsupervised learning models for clustering analysis. The performance of the different existing techniques, the pertinence ratio of the data involved, the effectiveness of the quality of the results obtained, analyzing accuracy and tests of positive and negative diagnoses, among others, will be evaluated.

Parallelly a distance profile will be determined between the mean profile of healthy individuals' groups formed according impact factors on protein profile. This approach will allow the identification of the molecular mass corresponding to the peaks that demonstrate to be different. A threshold will be set at half peak height to identify these intervals. This step will allow the association of potential proteins in these ranges using saliva protein database SalivaTecDB (http://salivatec.viseu.ucp.pt/salivatec$\mathrm{db})$. The area of each peak in each profile will be compared between all individual profiles, using a ttest, in order to identify peaks characteristic of each factor.

From these analyses will result intervals of molecular masses corresponding to proteins of interest (later confirmed by mass spectrometry).

\subsection{Construction of the Database for the Storage of SalivaPrints}

Protein profiles representing different conditions will result from this work and will be gathered in SalivaPrint database. Each SalivaPrint will be linked to the metadata collected in the questionnaires developed in 3.1. Tools will be developed to categorize, edit, visualize and export the data obtained to different formats, creating the necessary conditions for its use in the development of computational strategies for analysis and comparison of saliva protein profiles for diagnostic purposes. 


\section{PRELIMINARY RESULTS}

The implementation of the proposed strategy (Figure 1) has so far yielded the results described below.

So far it has been possible to collect 79 samples meeting the defined inclusion criteria (Table 1) and the metadata associated with each sample using the questionnaire developed by us.

Table 1: Number of saliva samples collected form healthy individuals after applying the selection criteria.

\begin{tabular}{|c|c|c|c|c|}
\hline & & \multicolumn{2}{|c|}{ Gender } & \multirow{2}{*}{ Total } \\
\hline & & Female & Male & \\
\hline \multirow{3}{*}{ 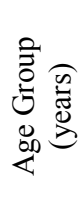 } & $<13$ & 12 & 4 & 24 \\
\hline & $13-24$ & 14 & 10 & 39 \\
\hline & $25-50$ & 22 & 17 & 16 \\
\hline \multicolumn{2}{|c|}{ Total } & 48 & 31 & 79 \\
\hline
\end{tabular}

A protein profile for each gender (Female $n=48$; Male $n=31$ ) was built (Figure 2 a), according to the methodology described in the section 3.3.
In order to determine how gender impact, the saliva total protein profile in health individuals (Health-SalivaPrint) a Kruskal-Wallis hypothesis test was applied (Figure 2). In top graph we could see the protein profiles from Female (red) and Male (blue) individuals. When Kruskal-Wallis test is applied, on lower graph the higher peak (green) with lower $p$ value (yellow) corresponds to molecular weight where it exists a significant difference between genders, so the gender characteristic signature.

A distance profile for each gender was built according to the methodology described earlier. The comparison of the mean-profile obtained from saliva of Female and Male individuals was used to establish a distance-profile (Figure 3). This distance profile reflects the molecular weight (MW) range corresponding to the proteins that are altered between genders. The creation of the distance profile allows the identification of the specific molecular mass according to different expressed peaks on each group (Figure 3).

The t-test applied on half-peak areas of all profiles showed the main differences were found in 2 of the typical 6 peaks of the saliva protein profile. Within these peaks, the MW ranges of peak 5 (58.5 - 63.2 $\mathrm{kDa})$ and peak $6(66.8-74.2 \mathrm{kDa})$.
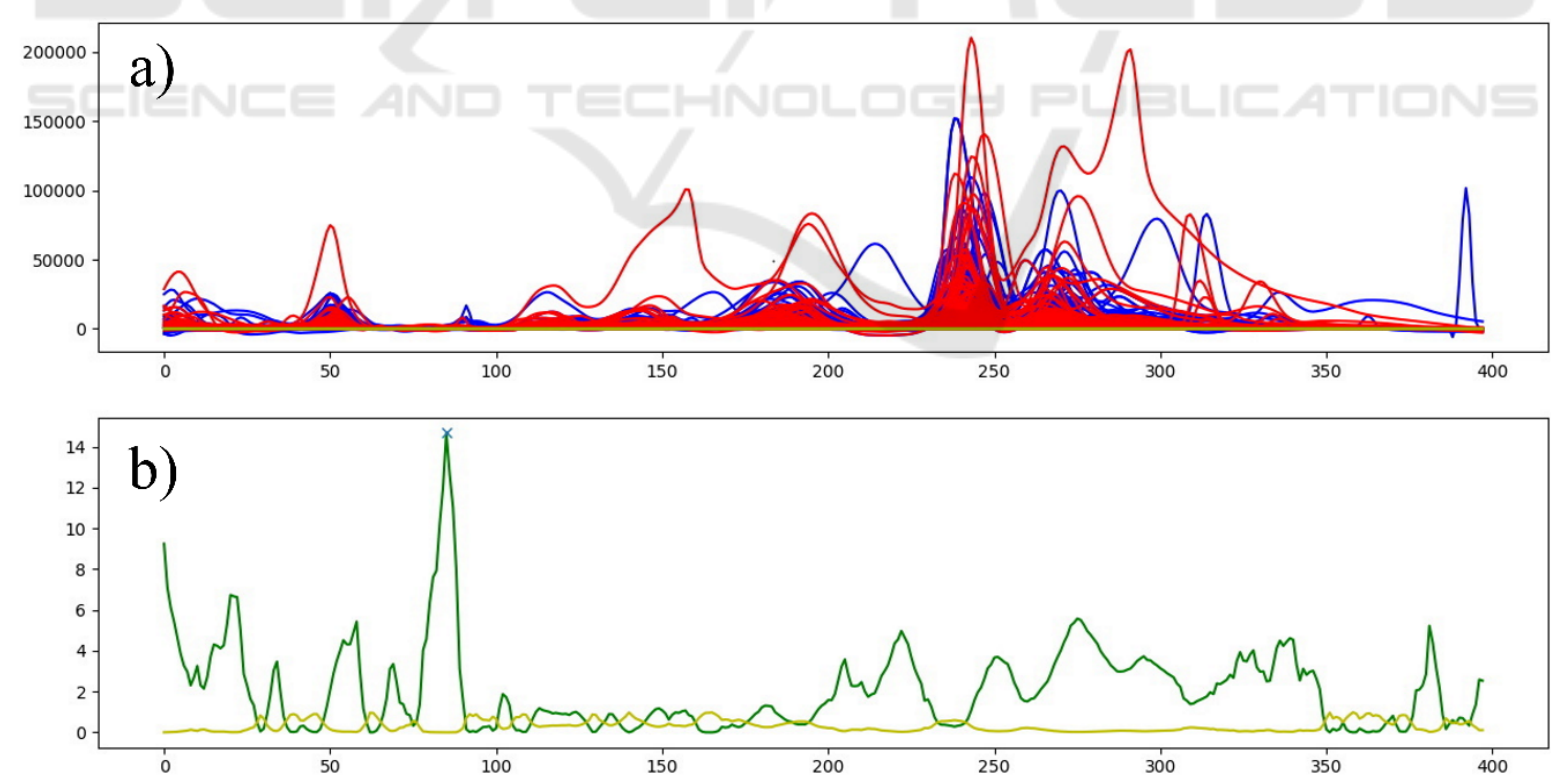

Figure 2: Application of the Kruskal-Wallis hypotheses test in determining profile signatures for gender identification. In a), red lines correspond to the profiles of female individuals and blue lines to the profiles of male individuals. In b) the green line corresponds to the test value and the yellow line to the $p$ value. Higher hypothesis test value (green) and lower $p$ value (yellow) correspond to the molecular weight were exist a clear difference on fluorescence between genders. 


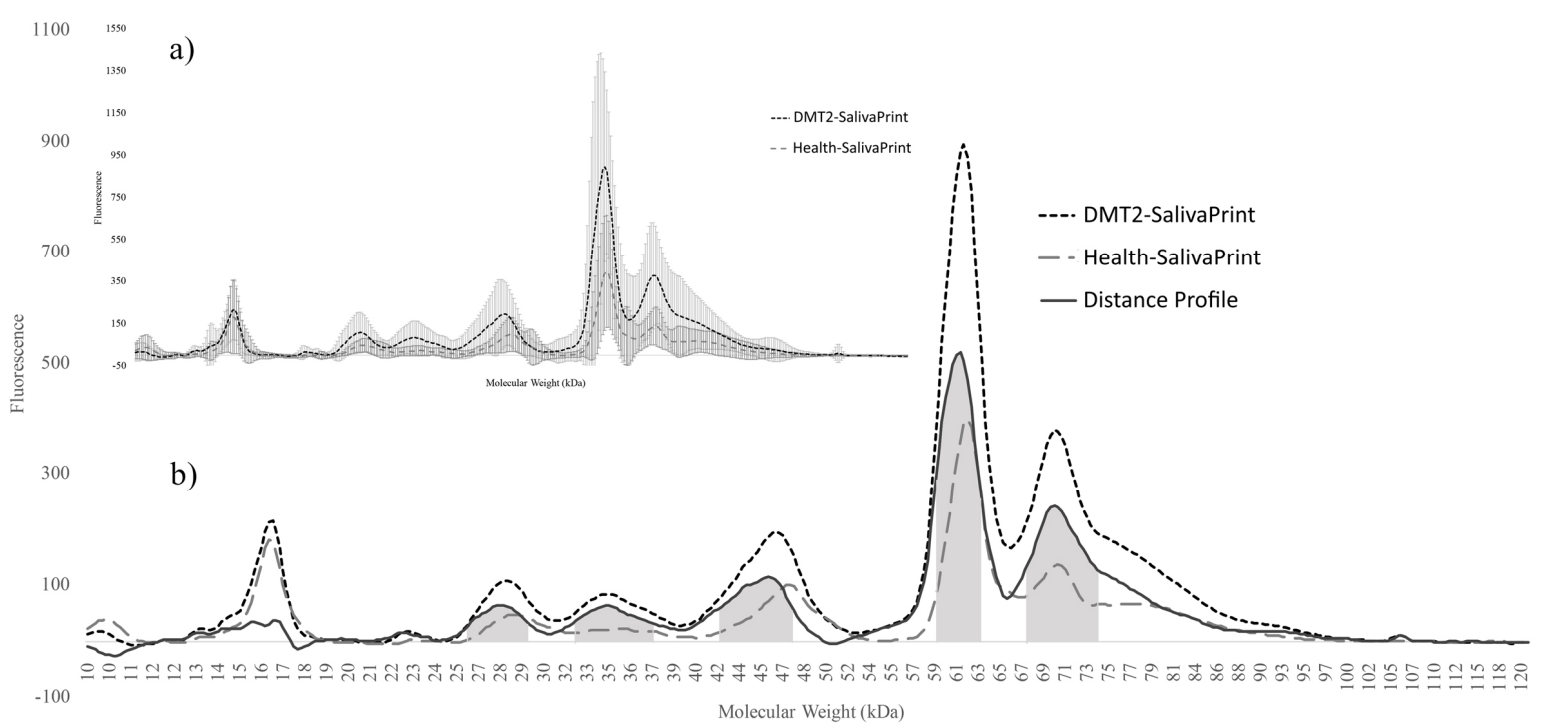

Figure 3: Protein profile distribution in Female (---) and Male (- - ) individuals. a) Female and Male mean-profile corresponding to the average of fluorescence per molecular weight. The standard deviations are represented by the vertical error lines. b) Protein profile for Female (----) and Male (-- ) and the distance-profile (-). Shaded areas correspond to the MW range of the proteins most altered between genders.

Once the distance profile and the respective MW ranges are established, it is possible, use SalivaTecDB (Figure 4), to associate potential proteins with molecular mass within the defined ranges and after that confirmed by mass spectrometry.

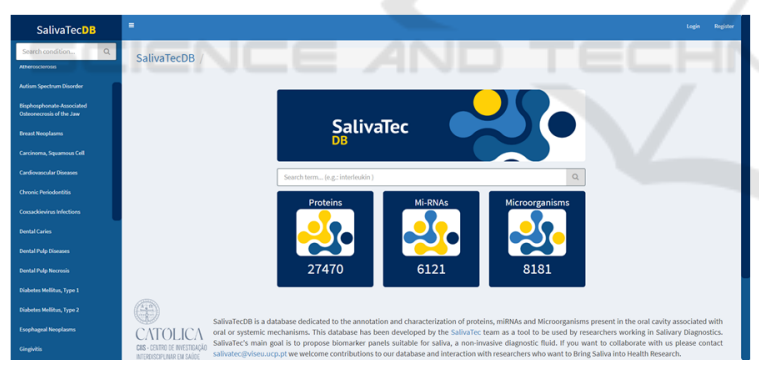

Figure 4: SalivaTecDB homepage. SalivaTecDB is dedicated to the annotation and characterization of proteins, miRNAs and Microorganisms present in the oral cavity, associated with oral or systemic mechanisms. This database was developed as a tool to be used by researchers working in Salivary Diagnostics.

\section{FUTURE WORK}

Currently we are developing the Python scripts for: data acquiring (from electrophoresis system) and treatment (profile alignment, peak determination and peak area calculation). These scripts will allow the total protein profile comparing between individuals and groups, with peak areas for statistical analysis, as well as analysis with machine learning strategies. The application of machine learning, for example Random Forest based on unsupervised models, allow the clusterization according to impact factors on profile (if it's the case) and thus define a characteristic Health-SalivaPrint.

\section{CONCLUSIONS}

This paper presents the SalivaPrint strategy and preliminary results already obtained with this approach. This work already allowed us to develop the methodologies that permits to obtain the total protein profile of health individuals (in a convenience sample). Through the analysis of these profiles it is possible to associate the potential proteins more representative of the differences between them using SalivaTecDB. The application of the Kruskal-Wallis test is a first approach on the identification of signatures, although there still need for other analysis to confirm the results.

Future work will be directed to python scripts development to acquire aligned protein profiles and determine the factors that influence the profile, as well as establish the machine learning strategy for cluster determination and its association to database (SalivaPrintDB).

It is expected with this work to use the database with SalivaPrints associated with an unsupervised machine learning algorithm that allows protein 
patterns recognition through profiles and personal data, without defined diagnosis, pathology or health situations. This approach will consider the morphology of the protein profile obtained with capillary electrophoresis in an automated manner and the influencing factors of saliva proteome, namely, the total protein concentration, age, gender and interindividual and intra-individual variability. These variables will be analyzed through the application of supervised analysis models in a first phase to identify the influence of each factor in the profile. Later with a higher number of individuals we resorted to unsupervised analysis models.

For this strategy to be used for diagnosis purposes, it is necessary to compare HealthSalivaPrint with a Disease-SalivaPrint. Since differences in saliva protein profiles have been observed in healthy people, depending on age and gender, it is important to take this into account in order to isolate the effect of the disease from the effects of these parameters.

This type of work is essential to find less invasive forms of diagnosis that take into account all the molecular and physiological variability of the individual.

\section{ACKNOWLEDGEMENTS}

Thanks are due to FCT/MCTES, for the financial support of Centre for Interdisciplinary Research in Health (UID/MULTI/4279/2019). Thanks, are also due to FCT and UCP for the CEEC institutional financing of $\mathrm{AC}$ Esteves.

\section{REFERENCES}

Anón. 2002. «Periodontal Screening and Recording (PSR) Index: precursors, utility and limitations in a clinical setting - Landry - 2002 - International Dental Journal Wiley Online Library». Obtido 24 de Março de 2019.

Arrais, Joel P., Nuno Rosa, José Melo, Edgar D. Coelho, Diana Amaral, Maria José Correia, Marlene Barros, e José Luís Oliveira. 2013. «OralCard: A bioinformatic tool for the study of oral proteome». Archives of Oral Biology 58(7): 762-72.

Castagnola, M., P. M. Picciotti, I. Messana, C. Fanali, A. Fiorita, T. Cabras, L. Calò, E. Pisano, G. C. Passali, F. Iavarone, G. Paludetti, e E. Scarano. 2011. «Potential applications of human saliva as diagnostic fluid». Acta Otorhinolaryngologica Italica 31(6):347-57.

Castagnola, M., E. Scarano, G. C. Passali, I. Messana, T. Cabras, F. Iavarone, G. Di Cintio, A. Fiorita, E. De Corso, e G. Paludetti. 2017. «Salivary biomarkers and proteomics: future diagnostic and clinical utilities». Acta Otorhinolaryngologica Italica 37(2):94-101.

Cruz, Igor, Eduardo Esteves, Mónica Fernandes, Nuno Rosa, Maria José Correia, Joel P. Arrais, e Marlene Barros. 2018. «SalivaPRINT Toolkit - Protein profile evaluation and phenotype stratification.» Journal of proteomics 171:81-86.

Formiga, F., M. Camafort, e F. J. Carrasco Sánchez. 2019. «Insuficiencia cardiaca y diabetes: la confrontación de dos grandes epidemias del siglo xxi». Revista Clínica Española.

Kaczor-Urbanowicz, Karolina Elżbieta, Carmen Martin Carreras-Presas, Katri Aro, Michael Tu, Franklin GarciaGodoy, e David TW Wong. 2017. «Saliva diagnostics Current views and directions». Experimental Biology and Medicine 242(5):459-72.

Kaushik, Ajeet e Mubarak A. Mujawar. 2018. «Point of Care Sensing Devices: Better Care for Everyone». Sensors (Basel, Switzerland) 18(12).

Loo, J. A., W. Yan, P. Ramachandran, e D. T. Wong. 2010. «Comparative Human Salivary and Plasma Proteomes». Journal of Dental Research 89(10):1016-23.

Mozaffari, Hamid Reza, Roohollah Sharifi, Asad VaisiRaygani, Masoud Sadeghi, Samad Nikray, e Rozita Naseri. 2019. «Salivary Profile in Adult Type 2 Diabetes Mellitus Patients: A Case-Control Study». J Pak Med Assoc 69(02):5.

Murr, Annette, Christiane Pink, Elke Hammer, Stephan Michalik, Vishnu M. Dhople, Birte Holtfreter, Uwe Völker, Thomas Kocher, e Manuela Gesell Salazar. 2017. "Cross-Sectional Association of Salivary Proteins with Age, Sex, Body Mass Index, Smoking, and Education». Journal of Proteome Research 16(6):2273-81.

Rosa, Nuno, Maria José Correia, Joel P. Arrais, Pedro Lopes, José Melo, José Luís Oliveira, e Marlene Barros. 2012. «From the Salivary Proteome to the OralOme: Comprehensive Molecular Oral Biology». Archives of Oral Biology 57(7):853-64.

Rosa, Nuno, Jéssica Marques, Eduardo Esteves, Mónica Fernandes, Vera M. Mendes, Ângela Afonso, Sérgio Dias, Joaquim Polido Pereira, Bruno Manadas, Maria José Correia, e Marlene Barros. 2016. «Protein Quality Assessment on Saliva Samples for Biobanking Purposes». Biopreservation and Biobanking 14(4):28997.

Schuller, Annemarie A. e Dorthe Holst. 2001. «Oral Status Indicators DMFT and FS-T: Reflections on Index Selection». European Journal of Oral Sciences 109(3):155-59.

The American Diabetes Association. 2019. «Classification and Diagnosis of Diabetes: Standards of Medical Care in Diabetes-2019». Diabetes Care 42 (Supplement 1):S13-28.

Wang, Xiaoqian, Karolina Elżbieta Kaczor-Urbanowicz, e David T. W. Wong. 2017. "Salivary Biomarkers in Cancer Detection». Medical oncology (Northwood, London, England) 34(1):7.

Wang, Zhihui, Yanyi Wang, Hongchen Liu, Yuwei Che, Yingying $\mathrm{Xu}$, e Lingling E. 2015. «Age-Related Variations of Protein Carbonyls in Human Saliva and Plasma: Is Saliva Protein Carbonyls an Alternative Biomarker of Aging?» $A G E$ 37(3). 